\title{
A Novel Approach for Security Analysis using Shift Factors for Limited Synchrophasor Observability
}

\author{
Backer Abu-Jaradeh \\ University of Southern California \\ abujarad@usc.edu
}

\author{
Mohammed Beshir \\ University of Southern California \\ beshir@usc.edu
}

\begin{abstract}
The adoption of synchrophasor technology has increased rapidly in the past decade. Many system operators have made synchrophasor applications available to operators, to reveal hidden operating conditions, and increase grid resiliency. The development of Linear State Estimation provided an innovative method to solve system states linearly at a faster rate, and serve as a backup to EMS should the conventional State Estimator fail to solve. Advanced applications were developed to take advantage of LSE solution to provide operators with alternative contingency analysis applications using synchrophasors data [6]. However, currently explored applications are presumed to run iteratively every couple of minutes, and therefore not taking advantage of high resolution of measurements available in synchrophasors. This work proposes a method to monitor system limits by leveraging linearization methods for contingency analysis, to better utilize the benefits of synchrophasors. Also, a practical approach is proposed to handle lack of full observability, to ensure tool operability with the industry infrastructure.
\end{abstract}

\section{Introduction}

The increase in load demand and the limited upgrades to transmission systems are pushing power systems to operate closer to their reliability boundaries, which may cause unforeseen operating conditions leading to cascading outages, uncontrolled system separation, or even blackouts. Therefore, the modern operation of the power grid requires continuous monitoring of System Operating Limits for normal and outage conditions, to ensure the reliable and secure operation of the system. Tools such as Real-Time Contingency Analysis (RTCA) are becoming a critical component of the modern power system operations environment to provide operators with the outlook necessary to guarantee that the system is operated within its pre-set boundaries should unforeseen outages happen. Power systems are becoming heavily dependent on advanced applications to reveal system conditions that were once unobservable by operators. Energy Management Systems (EMS) are equipped with comprehensive applications nowadays to help operators monitor the health of the grid, and even observe areas of the system that are not metered. State Estimator (SE) is an application within EMS that uses SCADA measurements and an iterative non-linear engine to provide downstream applications with a fully observable error-free system snapshot, for system monitoring and analysis. State Estimation is resource intensive by nature given the necessity to solve an optimization problem in an iterative fashion, which requires substantial amount of time to solve. Real-time network applications such as Real-Time Contingency Analysis use State Estimator solved powerflow cases to conduct iterative analysis to foresee system conditions should unscheduled outages occur in the power system. Real-Time Contingency Analysis usually handles thousands of contingencies, which is also resource intensive and time exhausting. In addition, typical RTCA runs iteratively every 3-5 minutes, and provides a list of potential violations for any possible contingency. Phasor Measurement Units (PMUs) enabled Linearization of the State Estimation problem, leading to the development of Linear State Estimator (LSE). LSE algorithms are presented in [1][4-10]. LSE provides solution at synchrophasor rate, which allows more visibility to the power grid as compared to conventional State Estimation [1][4-5]. With access to more system snapshots through Linear State Estimation, synchrophasor measurements can be used to enhance RTCA by filling in the gaps in the solutions using SCADA-based applications. This work investigates methods for using synchrophasor-based technology, vetted by Linear State Estimator to provide higherresolution results for Security Analysis, to reveal not only violations at multi-minute interval, but also 
dynamics of violations at synchrophasor rate. This work addresses practical considerations regarding system observability, which is driven by PMU deployments, and proposes a solution to overcome limitations caused by limited PMU coverage.

\section{Use of Linear Shift Factors (LSFs)}

Real-Time contingency analysis requires solving iterative powerflow equations for thousands of system topological scenarios to reveal potential exceedances to System Operating Limits (SOLs) following unscheduled loss of equipment in real-time. Multiple approaches have been proposed to perform security analysis using synchrophasor measurements [2]. However, these methods suggest a contingency analysis that runs iteratively over a multi-minute window, like SCADA-based applications. Although such applications might provide a backup solution to existing contingency analysis applications should they fail in real-time, they disregard the benefit of high-resolution measurement availability in synchrophasor data. Synchrophasors offer synchronized phasor measurements at rates of at least 30 samples per second (for $60 \mathrm{~Hz}$ systems), which allow monitoring of system dynamics and transients. Linear Shift Factors (LSF) provide a linear estimation to the security analysis problem, which allow much faster calculations as compared to solving powerflow iteratively [12]. Shift Factors are constants that can be used to estimate the powerflow on a specific transmission equipment, knowing the power on another. Two types of Linear shift factors are well studied in literature [3][12]; Power Transfer Distribution Factors (PTDFs) and Line Outage Distribution Factors (LODFs). Power Transfer Distribution Factor is the change in power flow on a transmission line relative to a power transfer between two buses (injection and withdrawal of power at two buses). [12]. PTDFs have the following mathematical definition:

$$
\operatorname{PTDF}_{a, b, l}=\frac{\Delta f_{l, a, b}}{\Delta P_{a, b}}
$$

Where,

$P T D F_{a, b, l}=$ Power Transfer Distribution Factor for Line $l$, given an injection and withdrawal at buses $a$ and $b$ respectively.

$\Delta f_{l, a, b}=$ Change in Line $l$ power flow for the transaction at buses $a$ and $b$.

$\Delta P_{a, b}=$ Power transferred in from bus $a$ to bus $b$

Line Outage Distribution Factors are constants that represent the estimated change of power on a transmission line or a transformer relative to a loss of another line [12]. Like PTDFs, LODF are defined as follows:

$$
L O D F_{l, c}=\frac{\Delta f_{l}}{f_{c}^{0}}
$$

Where,

$L O D F_{l, c}=$ Line Outage Distribution Factor for Line $l$, after the contingency of Line $c$

$\Delta f_{l}=$ Change in Line $l$ power flow following the contingency of Line $c$

$f_{c}^{0}=$ Power flow of contingency element $c$ before it was removed

\section{Security Analysis Using Shift Factors}

The objective of the proposed methodology is to provide real-time operators and engineers with fast realtime security analysis that uses synchrophasor measurements and Linear State Estimation results. Synchrophasor measurements provide accurate and fast measurements to voltage and current phasors, which can be used to provide accurate contingency analysis results. However, the relatively low number of synchrophasors available from the grid makes it challenging to observe the real-time state of the whole system, even when violations are localized to a relatively small electrical area. The deployment of PMUs has been focused on key substations of the transmission grid, such as heavily interconnected substations, or stations with intertie lines, without much deployment in locations to optimally target full system observability. In addition, with synchrophasors focusing on wide area monitoring and situational awareness, the deployment of PMUs has been spread out in the transmission systems. This paper describes a practical method to use Linear Shift Factors with a limited available PMU coverage in the system, to estimate and monitor System Operating Limits exceedances at the synchrophasor rate. Figure 1 below represents a generic 5-bus power system, which will be used to explain the methodology proposed in this paper.

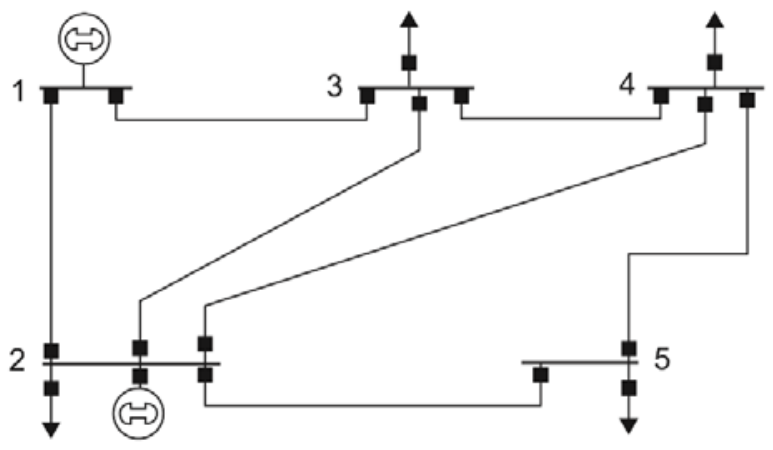

Figure 1. Generic 5-Bus Power System

Table 1 below shows the line loading information for all transmission lines in the case. For this example, 
we will attempt to estimate the post-contingency line flows for all lines, following the loss of line 2-3. PTDFs for all lines relative to the injection/withdrawal of power at buses 2 and 4 respectively are also shown. Buses 2 and 4 represent the main source and sink of the system, which in result will derive the highest PTDFs set for all major lines. Also, LODFs for all lines are provided for the contingency of line 2-3.

Table 1. MW, PTDFs for transaction between buses 2 and 4, and LODFs of the outage of line 2-3

\begin{tabular}{|c|c|c|c|c|}
\hline$l$ & $L_{l}^{\text {pre }}$ & $\% P T D F_{2,4, l}$ & $\% L O D F_{l, 2-3}$ & $L_{l, 2-3}^{\text {post }}$ \\
\hline $1-2$ & 79 & $-17.14 \%$ & $-33.3 \%$ & 60.02 \\
\hline $1-3$ & 62.14 & $17.14 \%$ & $33.3 \%$ & 80.98 \\
\hline $2-3$ & 57.16 & $28.57 \%$ & $-100 \%$ & 0 \\
\hline $2-4$ & 52.35 & $36.19 \%$ & $44.4 \%$ & 77.31 \\
\hline $2-5$ & 111.27 & $18.10 \%$ & $22.2 \%$ & 123.65 \\
\hline $3-4$ & -27.7 & $45.71 \%$ & $-66.7 \%$ & -66.02 \\
\hline $4-5$ & 16.13 & $18.10 \%$ & $-22.2 \%$ & 3.35 \\
\hline
\end{tabular}

With all the powerflow information readily available, we can use LODFs to estimate the postcontingency flow on Line $l$ following the loss of transmission line $k$ using the below equation [12] :

$$
\hat{f}_{l, c}=f_{l}^{0}+L O D F_{l, c} \times f_{c}^{0}
$$

Where, $\hat{f}_{l}$ is the power flow on Line $l$ after the removal of Line $c$

Estimations of the post-contingency line flows for the loss of Line 2-3 are presented in table 1 as well. Figure 2 presents the actual MW flow of the line following the contingency. The results agree with the estimated results as expected.

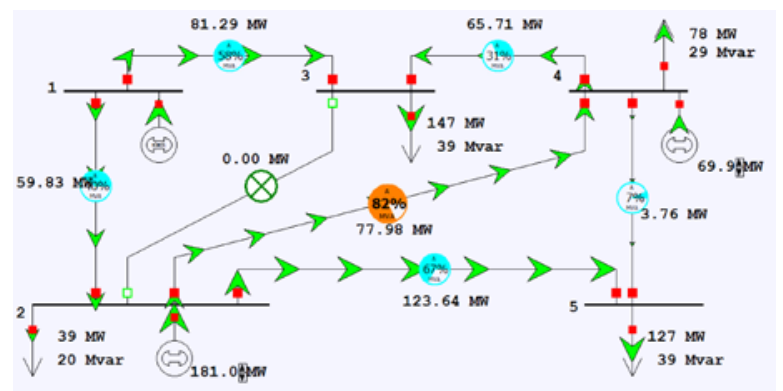

Figure 2. Simulation of the loss of Line 2-3 on the 5bus generic case in Figure 1

For real-time applications implementation, equation (3) can be fed with real-time measurements continuously to update the post-contingency flow at the synchrophasor rate, and provide an updated outlook to system operators on the monitored SOL, without the need to continuously solve full powerflow. $f_{l}^{\text {pre }}$ and $f_{c}^{\text {pre }}$ represent the real-time measurement of the limiting element (monitored element) and the contingency element prior to the loss of the line respectively. Therefore, the linear equation above can be used to estimate the post-contingency line flow for any transmission line, given the flow-measurement availability of the limiting and contingency elements. To apply that, equation (3) becomes,

$$
\hat{f}_{l, c_{\text {new }}}=f_{l_{\text {new }}}^{0}+L O D F_{l, c} \times f_{c_{\text {new }}}^{0}
$$

Where new denotes to the updated measurement for the line flow, as compared to the original flow from the solved powerflow.

The challenge of making use of LODFs with synchrophasor measurements resides in the lack of availability of PMUs at every substation of the system to provide full observability, that is necessary to solve the linear equations. This means that $f_{l_{\text {new }}}^{0}$ and $f_{c}^{0}$ new in equation (4) (the updated limiting and contingency elements flow measurements) might not be always available. Therefore, a practical methodology needs to be implemented to provide awareness for all contingencies and limiting elements, given the lack of measurement availability.

It is assumed that measurements at much lower rates are available from EMS or State Estimator cases through conventional methods. Since most State Estimators run at a rate of at least once every 5 minutes, a value for unobservable PMU locations is available as a reference, which is used as a reference flow, and updated using synchrophasor measurements. And since this value is used as a reference and gets updated with new measurements, it is referred to as $f_{\text {old }}$.

Let us define a new sensitivity factor called Line Flow Distribution Factor (LFDF), that is derived from two lines PTDFs as the following,

$$
L F D F_{l, k}=\frac{P T D F_{a, b, l}}{P T D F_{a, b, k}},
$$

Substituting (1) into (4) gives,

$$
L F D F_{l, k}=\frac{\Delta L_{l, a, b}}{\Delta L_{k, a, b}},
$$

From this equation, it can be observed that the relationship between the change of flow on two lines is constant and does not depend on the power injected in the power transfer transaction. Therefore, if line $l$ is not observed, we can use equation 5 to estimate the change in flow on Line $l$ using another observed Line $k$. Rewriting equation (5), and dropping the transaction index, we get,

$\Delta f_{l}=\Delta f_{k} \times L F D F_{l, k}$,

Or, 


$$
\left(f_{l_{\text {new }}}-f_{l_{\text {old }}}\right)=\Delta f_{k} \times L F D F_{l, k},
$$

Rearranging,

$$
f_{l_{\text {new }}}=f_{l_{\text {old }}}+\Delta f_{k} \times L F D F_{l, k} \text {, }
$$

Similarly, assuming $f_{c \text { new }}^{0}$ (the updated contingency element power flow before the contingency) is not observable, it can be written in terms of another line flow $f_{j}$ which is observable. Applying equation (7) to equation (4), we get,

$$
\begin{aligned}
\hat{f}_{l, c_{\text {new }}}= & f_{l_{\text {old }}}^{0}+\Delta f_{k}^{0} \times L F D F_{l, k}+ \\
& +L O D F_{l, c} \times\left[f_{c_{\text {old }}}^{0}+\Delta f_{j}^{0} \times L F D F_{c, j}\right]
\end{aligned}
$$

Equation (8) allows the estimation of post contingency loading on line $l$ for the contingency of Line $c$, without the necessity of having updated measurements from Lines $l$ and $c$. This provides system operators with awareness of the system conditions for parts of the system that in not otherwise observable. This method is also applicable to reactive-power calculations, although the focus here is on real power since most line overloads in power systems are caused by high real power flow. Pre-calculating LFDFs allows a very fast procedure to scan the system for possible violations for all possible contingencies. In addition, the linear nature of the equations enables performing the calculations at synchrophasor rate. Furthermore, the key system assumptions are:

(1) The system model and enough telemetry are available to calculate the sensitivity factors. Once the factors are calculated, they are assumed to be valid and correct as long as the system did not go through a major change that affects these factors, such as a significant topology change or a major generation profile change. This assumption is valid given that those changes are monitored and flagged in real-time to trigger the recalculation of all sensitivity factors.

(2) This method applies to single and multiple contingencies, given that specific multiple contingencies are pre-defined, and the corresponding sensitivity factors for the multiple contingencies are precalculated. Therefore, a separate set of sensitivity factors are required for multiple outages scenarios. This assumption is also valid since system operators are interested in monitoring only credible multiple contingencies, such as lines sharing transmission towers, right of ways, or critical transmission paths.

(3) The sensitivity factors are calculated with an injection of power relatively far from the reference bus, to ensure the accuracy of the calculated factors, without the slack bus contributing to reverse the flow. An example of apply this method is presented in the following part of this section.

Let us consider a modified 5-bus system case, where only measurements at buses 1 and 4 are available. Also, loads were modified little to simulate a change over time in lines loading. Updated case information is

\begin{tabular}{|c|c|c|c|}
\hline Line $l$ & $f_{l_{\text {old }}}^{0}$ & $f_{l_{\text {new }}}^{0}$ & $\Delta f_{l}$ \\
\hline $2-3$ & $57.16 \mathrm{MW}$ & \multicolumn{2}{|c|}{ Unobservable Contingency } \\
\hline $2-4$ & $52.35 \mathrm{MW}$ & $53.67 \mathrm{MW}$ & 1.32 MW \\
\hline $2-5$ & 111.27 MW & \multicolumn{2}{|c|}{$\begin{array}{c}\text { Unobservable Limiting } \\
\text { Element }\end{array}$} \\
\hline $4-5$ & 16.13 MW & 17.20 MW & $1.07 \mathrm{MW}$ \\
\hline
\end{tabular}
presented in table 2 below.

Table 2. Updated MW flow with Lines only connected to substations 1 and 4

In order to estimate the post-contingency flow on Line 2-5 for the contingency of Line 2-3 for example, the equation becomes,

$$
\begin{gathered}
\hat{f}_{2-5,2-3_{\text {new }}}=f_{2-5} \text { old }_{\text {old }}^{0}+\Delta f_{4-5} \times L F D F_{2-5,4-5}+ \\
+L O D F_{2-5,2-3} \times\left[f_{2-3}\right. \text { old } \\
\left.\Delta f_{2-4} \times L F D F_{2-3,2-4}\right]
\end{gathered}
$$

This provides a post contingency estimation of 128.61 MW for the limiting element 2-5, for the loss of the line 2-3. The result can be compared with the powerflow simulation result shown below in Figure 3. The resulting error in estimation is $0.4 \%$.

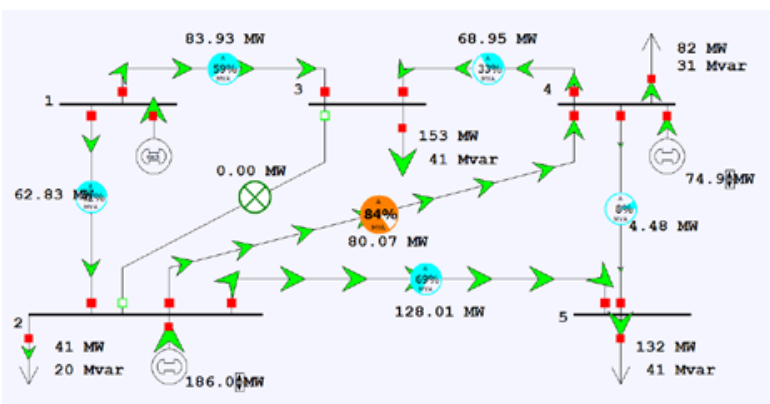

Figure 3. Simulation of the loss of Line 2-3 on the updated 5-bus generic case in Figure 1

\section{Case Study and Simulations}

A case study is presented in this section to evaluate and demonstrate the numerical accuracy of the proposed methodology. The case uses the IEEE 39 bus system, which is reduced from New-England's power system model. This case study illustrates the methodology to perform linear security analysis using synchrophasor measurements by taking advantage of distribution factors to estimate the post contingency 
flow on transmission equipment where observability is not present. To demonstrate the numerical viability of this approach, the results are compared to the powerflow simulations using PowerWorld Simulator.

\subsection{IEEE 39-Bus System}

The IEEE 39 bus test system is a 10-machine test system model that is reduced from New-England Power System that has 10 generators and 46 lines [16][17]. A one line presentation of the power system model is shown in Figure 4 below. PMU observable transmission lines and buses are shown in red. Table 3 below shows the line loading information for some transmission lines in the case, as an example, we will attempt to estimate the post-contingency line flows for all lines, following the loss of line 2-3.

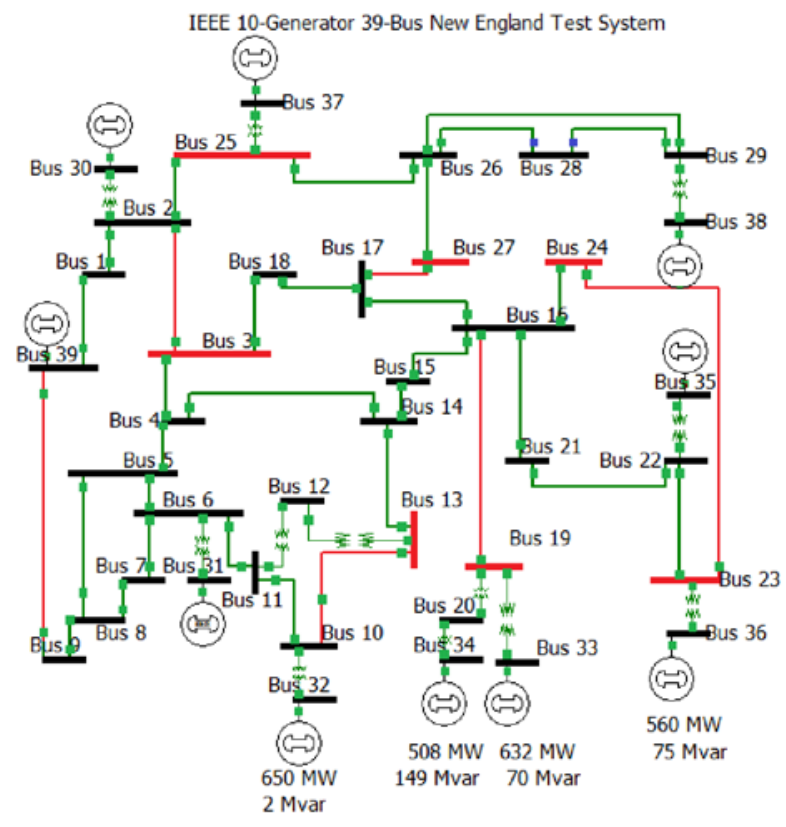

Figure 4. IEEE 39-Bus System with Limited PMU Observability

PTDFs for all lines relative to the injection/withdrawal of power at buses 25 and 9 respectively are also shown in table 3 . Similar to the previous exercise, buses 25 and 6 represent the main source and sink of the meshed transmission system, which in result will derive the highest PTDFs set for all major lines.

Table 3. MW, PTDFs for transaction on buses 25 and 6

\begin{tabular}{|c|c|c|}
\hline Line $l$ & $f_{l}^{\text {pre }}$ & $\%$ PTDF $_{25,6, l}$ \\
\hline $2-3$ & $364.72 \mathrm{MW}$ & $55.73 \%$ \\
\hline $2-25$ & $231.50 \mathrm{MW}$ & $75.78 \%$ \\
\hline $3-18$ & $34.09 \mathrm{MW}$ & $2.97 \%$ \\
\hline
\end{tabular}

\begin{tabular}{|l|l|l|}
\hline $17-27$ & $-11.51 \mathrm{MW}$ & $24.22 \%$ \\
\hline $26-27$ & $270.44 \mathrm{MW}$ & $24.22 \%$ \\
\hline
\end{tabular}

In order to demonstrate the numerical viability of the proposed method, we will attempt to calculate the post contingency flow of multiple transmission lines, following the loss of the line 2-25. As an example, to calculate the post-contingency flow on line 3-18, from equation (3) we get,

$\hat{f}_{3-18,2-25}=f_{3-18}^{0}+L_{O D F_{3-18,2-25}} \times f_{2-25}^{0}$

With a LODF of $73.6 \%$, the estimated postcontingency line flow of line 3-18 for the contingency of line 2-25 is equal to $204.08 \mathrm{MW}$. This is verified by simulation as shown in Figure 5, with an error in estimation of $0.6 \%$.

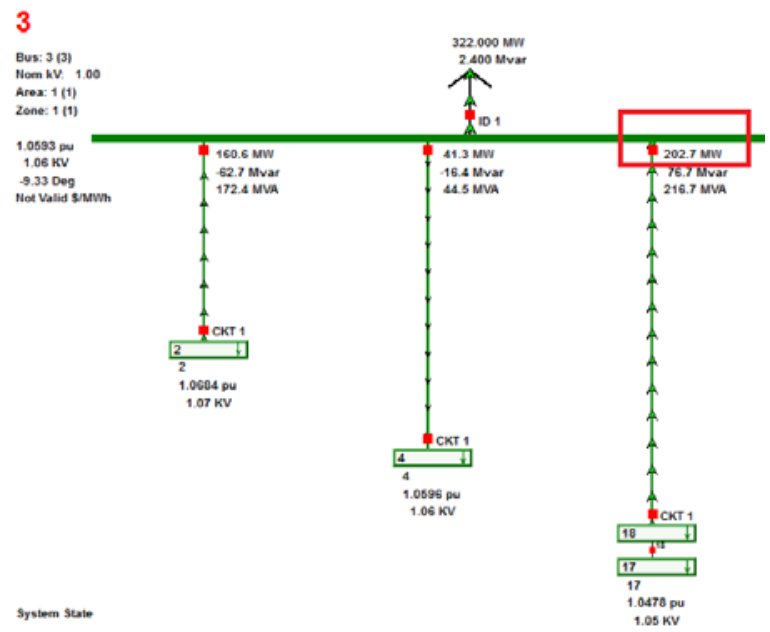

Figure 5. Post-Contingency flow of line 3-18 for the loss of line 2-25

Since both lines are not observable by PMUs, the change in pre-contingency line flow with time cannot be calculated as the updated flow is not known for both lines, due to lack of PMU observability. Using equation (8), we can rewrite equation (10) using other observable lines $k$ and $j$, in which equation (10) becomes as follows,

$$
\begin{aligned}
\hat{f}_{3-18,2-25_{\text {new }}=} & f_{3-18_{\text {old }}}^{0}+\Delta f_{k} \times L F D F_{3-18, k} \\
& +L O D F_{3-18,2-25} \times\left[f_{2-25, j} j_{\text {old }}\right. \\
& \left.+\Delta f_{j} \times L F D F_{2-25, j}\right]
\end{aligned}
$$

Let us assume that the system state changed a little, and the updated flows for the observable lines from table 3 are shown in table 4 . Similar to the previous exercise, loads were modified slightly to simulate a change over time in lines loading. 
Table 4. Updated MW flow with Lines only connected to substations 1 and 4

\begin{tabular}{|c|c|c|c|}
\hline Line $l$ & $f_{l_{\text {old }}}^{0}$ & $f_{l_{\text {new }}}^{0}$ & $\Delta f_{l}$ \\
\hline $2-3$ & $364.72 \mathrm{MW}$ & $378.4 \mathrm{MW}$ & $13.68 \mathrm{MW}$ \\
\hline $2-25$ & $231.50 \mathrm{MW}$ & \multicolumn{2}{|c|}{ Unobservable Contingency } \\
\hline $3-18$ & $34.09 \mathrm{MW}$ & \multicolumn{2}{|c|}{\begin{tabular}{|} 
Unobservable Limiting \\
Element
\end{tabular}} \\
\hline $17-27$ & -11.51 & $4.97 \mathrm{MW}$ & $16.48 \mathrm{MW}$ \\
\hline
\end{tabular}

From Figure 4, selecting line $17-27$ as line $k$, and line 2-3 as line $j$, and substituting in equation (11) we get,

$\hat{f}_{3-18,2-25_{\text {new }}}=f_{3-18} \mathrm{old}_{\text {old }}+$

$$
\begin{aligned}
& \Delta f_{17-27} \times L F D F_{3-18,17-27}+ \\
&+ L O D F_{3-18,2-25} \times\left[f_{2-25}{ }_{\text {old }}\right. \\
&\left.+\Delta f_{2-3} \times L F D F_{2-25,2-3}\right]
\end{aligned}
$$

Equation (12) calculates the Post Contingency Line flow of Line 3-18 for the loss of line 2-25 to be 218.29 MW. Verification using powerflow simulations has been performed and provides a postcontingency flow of $216.83 \mathrm{MW}$, with a tolerance of $0.67 \%$, as shown in Figure 6 .

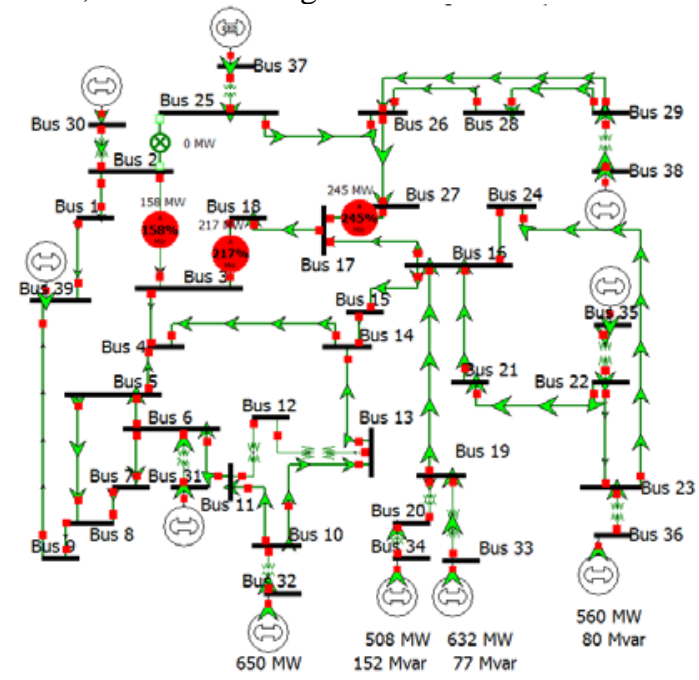

Figure 6. Post-Contingency flow of line 3-18 for the loss of line 2-25

Table 5 presents the Estimation of post contingency line flow for the major lines relatively close to the observable lines, and demonstrates the accuracy of the proposed approach. All the estimations are accurate with acceptable tolerances. It is worth mentioning that using this approach, a security analysis was performed only using a basecase and two PMUs, to monitor System Operating Limits, even for unobservable footprint. Table 5 shows the transmission lines surrounding the PMU observable lines, and other simulation information where,

Line $l$ - Transmission Line

$f_{l_{\text {old }}}^{0}$ - Base-case pre-contingency flows

$f_{l_{\text {new }}}^{0}$ - Updated base case pre-contingency flows

LODF - Line Outage Distribution Factor

$\hat{f}_{l_{\text {new }}}$ (Estimate) - Estimated post-contingency flows

$\hat{f}_{l_{\text {new }}}$ (Powerflow) - Actual post-contingency flows

$\%$ Error - Percentage errors in estimation

Table 5. Estimation and accuracy of PostContingency line flows for the loss of Line 2-25 with limited observability

\begin{tabular}{|c|c|c|c|c|c|c|}
\hline Line $l$ & $\begin{array}{c}f_{l_{\text {old }}}^{0} \\
(\mathrm{MW})\end{array}$ & $\begin{array}{c}f_{l_{\text {new }}}^{0} \\
(\mathrm{MW})\end{array}$ & LODF \% & $\begin{array}{l}\hat{f}_{l_{\text {new }}} \\
\text { (Estimate) }\end{array}$ & $\begin{array}{c}\hat{f}_{l_{\text {new }}} \\
\text { (Powerflow) }\end{array}$ & \% Error \\
\hline $2-3$ & 364.72 & 378.4 & 88 & 158.31 & 158.04 & 0.17 \\
\hline $2-25$ & 231.50 & & -100 & 0 & 0 & 0 \\
\hline $3-18$ & 34.09 & & 73.6 & 218.29 & 216.83 & 0.67 \\
\hline $17-18$ & 192.32 & & 73.6 & 376.52 & 376.30 & 0.58 \\
\hline $17-27$ & -11.51 & 4.97 & 100 & 239.59 & 245.37 & 2.36 \\
\hline $25-26$ & 78.61 & & 100 & 329.71 & 342.22 & 3.66 \\
\hline $26-27$ & 270.44 & & 100 & 521.54 & 530.75 & 1.74 \\
\hline
\end{tabular}

With such small system models, the tolerance typically is contributed to by the swing bus or the boundary buses as the external system is modeled with equivalent generators at the boundaries and the slack is picked up at the boundaries and at the swing bus. It is also noticeable that error percentage increases the further away the limiting element or the contingency element are from the observable branch that is used for the calculation. Therefore, more PMU deployments means increased accuracy of the estimations for contingency analysis results.

\section{Conclusions}

This paper proposes a methodology to perform security analysis using synchrophasor measurements and Linear State Estimation technology with limited observability on the power grid. This method increases the situational awareness of the system, to extend beyond the observable footprint of the grid, while maintaining a solution at the synchrophasor rate to provide visibility of system dynamics. The method produces accurate estimations representing the power grid following the loss of transmission lines and transformers, with a linear solution that solves as fast as PMU measurement rate. The accuracy of results depends on the validity of the sensitivity factors. Therefore, monitoring the system for changes affecting the factors to trigger re-calculation of LFDFs is crucial to guarantee accurate estimations. This applies to both single and multiple contingency definitions. It is also 
imperative to ensure that the reference bus, in the case used to calculate the sensitivity factors, is relatively far from the power injection, to ensure accurate calculations of these factors. The accuracy of results also depend on the PMU coverage of the system. Increase in PMU adoption allowing additional measurements across the system and leveraging Linear State Estimation to provide accurate data and expanded visibility to the security analysis, will provide higher accuracy of estimations.

\section{References}

[1] K. D. Jones, "Synchrophasor-Only Dynamic State Estimation \& Data Conditioning," 2013.

[2] N. Nayak, L. Zhang, K. Martin, I. Dobson, A. Bose and D. J. Sobajic, "Practical Implementation of Synchrophasor Based Real-Time Contingency Analysis," 2018 North American Power Symposium (NAPS), Fargo, ND, 2018, pp. 1-6.K.

[3] C. S. Song, C. H. Park, M. Yoon, G. Jang, "Implementation of PTDFs and LODFs for Power System Security,” Journal of International Council on Electrical Engineering, (2011), 1:1, 49-53, DOI: 10.5370/JICEE.2011.1.1.049

[4] D. Jones, J. S. Thorp, and R. M. Gardner, "Three-phase linear state estimation using phasor measurements," in Proc. IEEE Power Eng. Soc. General Meeting, Vancouver, BC, Canada, Jul. 21-25, 2013, pp. 1-5.

[5] L. Zhang, A. Bose, A. Jampala, V. Madani and J. Giri, "Design, testing, and implementation of a linear state estimator in a real power system,” IEEE Trans. Smart Grid., doi: 10.1109/TSG.2015.2508283.

[6] T. Yang, H. Sun, and A. Bose, "Transition to a two-level linear state estimator - Part II: Algorithm,” IEEE Trans. Power Syst., vol. 26, no.1, pp. 54-62, Feb. 2011.

[7] H. Chen, L. Zhang, J. Mo, and K. Martin, "Synchrophasor-Based Real-Time State Estimation and Situational Awareness System for Power System Operation,” J. Mod. Power Syst. Clean Energy (2016). doi:10.1007/s40565- 016-0212-9.

[8] L. Zhang, H. Chen, K. Martin, A. Faris, M. Vutsinas, T. Bradberry, E. Phillips, B. Abu-Jaradeh, J. Bui, "Successful Deployment and Operational Experience of Using Linear State Estimator in Wide-Area Monitoring and Situational Awareness Projects,” IET Generation, Transmission \& Distribution., vol. 11, no. 18, pp. 44764483, Dec 2017

[9] L. Zhang, H. Chen, N. Nayak, M. Vutsinas, T. Bradberry, E. Phillips, A. Faris "Benefit of Using Linear State Estimator for Synchrophasor Applications,” in Proc. IEEE Power Eng. Soc. General Meeting, Chicago, IL, USA, Jul. 17-21, 2017, pp. 1-5

[10]L. Zhang, H. Chen, K. E. Martin, and A. Faris, "Practical Issues of Implementation of Linear State Estimator in WECC”, IEEE PES Innovative Smart Grid Technologies Conferences (ISGT), Minneapolis,
September 2016

[11] Ian Dobson, Effect of network contingencies on a subnetwork, Iowa State University, dobson@iastate.edu, October 18, 2017

[12]A. Wood and B. Wollenberg, Power Generation, Operation, and Control, New York: Wiley, 1984

[13] V. Brandwajn, "Efficient bounding method for linear contingency analysis," in IEEE Transactions on Power Systems, vol. 3, no. 1,pp. 38-43, Feb 1988.

[14] V. Brandwajn and M. G. Lauby, "Complete bounding method for AC contingency screening," in IEEE Transactions on Power Systems, vol. 4, no. 2, pp. 724729, May 1989.

[15] R. D. Zimmerman, C. E. Murillo-Sanchez, and R. J. Thomas, "Matpower: Steady-State Operations, Planning and Analysis Tools for Power Systems Research and Education," Power Systems, IEEE Transactions on, vol. 26, no.1,pp.12\{19,Feb.2011.

[16]T. Athay, R. Podmore, and S. Virmani, "A Practical Method for the Direct Analysis of Transient Stability," IEEE Transactions on Power Apparatus and Systems, vol. PAS-98, no. 2, March/April 1979, pp. 573-584.

[17] Illinois Center for a Smarter Electric Grid (ICSEG), http://icseg.iti.illinois.edu/ieee39-bus-system/ 